\title{
Lactate production retards, not causes, acidosis: a theoretical approach for physical education students.
}

\author{
\{underlineHohl, R.; Silva, F.O.C.; Lazarim, F.L.; Tessutti, L.S.; Sarraipa, M.; Macedo, D.V.
}

Laboratório de Bioquímica do Exercício LABEX, Departamento de Bioquímica, Instituto de Biologia

UNICAMP, Campinas São Paulo Brasil.

The widespread belief that intense exercise causes the production of lactic acid that contributes to acidose is erroneous. This belief, carried out by physical education and other professionals, interferes on methods of training and raise the opinion that muscle lactate is the vilain of exercise fatigue. For a theorical approach we show a structural illustration of all the glycolysis reactions with enphasis to phosphoglycerate kinase reaction wich envolves a simple phosphate transfer from the first carbon of 1,3 bisphosphoglycerate to ADP, forming ATP. The carboxyl group of 3 phosphoglycerate remains unprotonated for the remaining intermediates of glycolisis. From this biochemical fact it is impossible the production of acid lactic causing the release of a proton. Actually, glycolysis releases 2 protons and lactate formation consumes 2 protons. After this biochemical explanation we present three illustrated exercises situations: low intensity, anaerobic threshold and high intensity exercise. From this point the students can understand that the protons came mainly from the ATP hydrolysis and when the ATP demand for muscle contraction is met by mithocondrial respiration, there is no proton accumulation in the cell as protons are used by mithocondria to maintain the proton gradient in the intermembranous space. When the ATP hydrolysis exceeds mithocondria buffering capacity and ATP demand is supplied by nonmithocondrial sources, protons increase inside the cell causing acidoses. Lactate production increases under these cellular conditions to prevent pyruvate accumulation and supply the NAD+ for glycolysis. Lactate also retards the acidoses by the symport with protons (from ATP hydrolysys not from lactate production) mediated by Lactate Transporters (MCT). Thus, increased blood lactate detection is the effect not the cause of acidosis. The students must understand that if muscles do not produce lactate, acidoses and fatigue would accour faster, impairing high intensity exercise performance. This information must be considered in their training schedules. 